\title{
Concepciones de niños y niñas sobre la inteligencia ¿Qué papel se otorga a las funciones ejecutivas y a la autorregulación?
}

\section{Children's Conceptions of Intelligence. What is role of Executive Functions and Self-regulation?}

\author{
Mónica M. Pino Muñoz \\ Universidad del Bío Bío, Chillán, Chile \\ ORCID: https://orcid.org/0000-0001-9551-3930 \\ Vanessa Arán Filippetti \\ Consejo Nacional de Investigaciones Científicas y Técnicas, Buenos Aires, Argentina \\ ORCID: https://orcid.org/0000-0002-0753-5089
}

Recibido 23-01-19 Revisado 05-02-19 Aprobado 23-04-18 En línea 26-04-19

*Correspondencia

Email:mpinom@ubiobio.cl
Citar como:

Pino Muñoz, M., \& Arán Filipetti, V. (2019) Concepciones de niños y niñas sobre la inteligenciąQué papel se otorga a las funciones ejecutivas y a la autorregulación?Propósitos y Representaciones, 7(2), 269303. doi: http://dx.doi.org/10.20511/pyr2019.v7n2.281 


\section{Resumen}

La inteligencia constituye un constructo de notable impacto en nuestro contexto sociocultural, evidenciado en un amplio desarrollo de definiciones conceptuales y operacionales, donde surgen aseveraciones que van desde privilegiar elementos de tipo cognitivo y abstracto, a elementos emocionales y sociales, hasta perspectivas que se enfocan en los procesos que subyacen al desempeño inteligente, como es el caso de las funciones ejecutivas (FE), las cuáles se configuran como procesos cognitivos que posibilitan la autorregulación (Miyake \& Friedman, 2012). De igual modo, existen enfoques que otorgan a la inteligencia un rol relevante y predictivo del rendimiento escolar, aspecto fortalecido por la perspectiva psicométrica donde el concepto de inteligencia se ha vinculado estrechamente con el aprendizaje. En esta línea, también encontramos la perspectiva clásica de inteligencia cristalizada de Cattell (1943), la cual hace alusión al conocimiento adquirido a través de procesos educativos y culturales. El objetivo de este estudio fue conocer las concepciones que niñas y niños, de edades comprendidas entre 8 y 12 años, otorgan al concepto de inteligencia, las características que le atribuyen y la valoración que hacen de las propias capacidades intelectuales. El análisis de los datos se llevó a cabo mediante la teoría fundamentada y los resultados evidencian principalmente que los participantes atribuyen al constructo de inteligencia componentes referidos al manejo de contenidos escolares y al rendimiento cuantitativo; además de poner en manifiesto elementos relacionados con las FE y la autorregulación no sólo en la significación que hacen del constructo, sino también al caracterizar y valorar el desempeño inteligente.

Palabras Clave: Inteligencia; Funciones ejecutivas; Autorregulación; Niños.

\section{Summary}

Intelligence is a construct of notable impact in our sociocultural context, related to a wide range of conceptual and operational definitions which originate positions that highlights cognitive and abstract elements and emotional and social skills, as well was perspectives that are focused on processes underlying the intelligent performance, such as in the case of executive functions (EFs), which are configured as cognitive processes that make it possible the self-regulation (Miyake \& Friedman, 2012). Similarly, there are approaches that give intelligence a relevant and predictive role of school performance, an aspect strengthened by the psychometric perspective, where the concept of intelligence has been closely linked to learning. In this line, we also find the classical perspective of crystallized Intelligence of Cattell (1943), which refers to the knowledge acquired through educational and cultural processes. The aim of this study is to know the conceptualizations of intelligence of children aged 8-12, the features they give to them, and the assessment of their own intellectual capacities. The data analysis was carried out through the grounded theory and the results mainly show that participants attribute components referred to the management of school contents and quantitative performance to intelligence. Moreover, the answers show elements related to EFs and self-regulation not only when considering the meaning of the construct, but also while characterizing and evaluating the intelligence performance.

Keywords: Intelligence; Executive Functions (EFs); Self-regulation; Children

\section{Introducción}

La conceptualización del constructo de inteligencia representa un trabajo exigente y de gran complejidad, además de constituir un aspecto relevante no sólo para la ciencia, sino también para las políticas públicas, orientando directrices educacionales, asistenciales y de salud (Marambio, Gil de Montes, Valencia \& Zubieta, 2015). Por otra parte, observamos una multiplicidad de definiciones conceptuales del término, definiciones que, a lo largo del tiempo, han experimentado cambios paradigmáticos que enriquecen el discurso y despiertan nuevos intereses disciplinarios (Ardila, 2011; Cabas-Hoyos, González-Bracamonte \& Hoyos-Regino, 2017; Rosas, Boetto \& Jordán, 2005). 
Si la conceptualización del término referido a la inteligencia resulta relevante para muchos estudiosos y significativo para el devenir de directrices que impactan la vida de los sujetos, resulta también importante interrogarnos acerca de las concepciones de este constructo que manejan niñas y niños de nuestros contextos educativos, cómo lo caracterizan e interpretan, los significados que le otorgan y las atribuciones que realizan a partir de este concepto. Desde la perspectiva de la Teoría Fundamentada (TF) se pretende conocer la teoría relativa a la inteligencia que emerge de sus opiniones, la que manejan en la cotidianidad de sus vivencias escolares y es parte central de los modelos explicativos que elaboran no sólo de sus propios procesos de aprendizaje (Bravo, 2012), sino también de aspectos afectivos, sociales y motivacionales relevantes en la construcción de propia la identidad (Toledo, 2012). Elementos centrales, donde el contexto escolar adquiere significancia, pues se constituye en un espacio físico y psicológico relevante y propicio para el desarrollo de las propias potencialidades y generación de recursos adaptativos (Sánchez, 2008).

\section{Inteligencia y cambios de paradigmas.}

La inteligencia viene concebida como un proceso cognitivo de alta complejidad en el cual intervienen diferentes habilidades y cuya conceptualización no se ha visto exenta de enfoques teóricos diferentes e incluso contradictorios (Ardila, 2011; Cabas-Hoyo et al., 2017; Pacheco, 2003). En la literatura, podemos encontrar visiones que se centran en la elaboración de definiciones teóricas que subrayan aspectos relacionados con habilidades abstractas, con la capacidad para resolver problemas, o bien con facultades relativas a la comprensión y al entendimiento (Isaza \& Calle, 2016; Rosas et al., 2005). Todas, perspectivas que destacan elementos cognitivos y abstractos, que caracterizan una conceptualización de inteligencia de gran fuerza en nuestros contextos culturales, influyendo en diferentes áreas, tales como el ámbito escolar, político y social (Rosas et al., 2005).

Diferencias y controversias, también encontramos en los enfoques que abordan temáticas relativas a la estabilidad o modificabilidad de las habilidades intelectuales. Específicamente, nos referimos a perspectivas teóricas que postulan a la base de la determinación de la inteligencia variables de tipo genético hereditario y, en su contraparte, variables de tipo ambiental y cultural (Rosas et al., 2005). Según estos enfoques, tendríamos dos perspectivas centrales a la hora de referirnos a la fijación de las habilidades intelectuales de las personas, la primera de ellas defiende una concepción hereditaria donde el límite intelectual viene dado por el potencial genético transmitido y la segunda postura aboga por el rol de las variables ambientales, culturales y socioeconómicas en la estimulación y desarrollo de la inteligencia, sobre todo en los primeros años de vida (Ardila, 2011; Herrnstein \& Murray, 1994; Rosas et al., 2005).

Otro aspecto importante de destacar lo constituye el hecho de que el estudio de la inteligencia en psicología, desde sus orígenes estuvo relacionado con la posibilidad de su medición (Ardila, 2011; Rosas et al., 2005). En efecto, la psicometría ha orientado muchos de sus esfuerzos en encontrar una medida válida de este constructo, utilizando en la mayoría de los casos muestras en los recintos escolares y mediándolo a través de los contenidos contemplados en sus currículos (Isaza \& Calle, 2016; Rosas et al., 2005). Desde esta perspectiva, no resulta difícil comprender los enfoques que vinculan la inteligencia, con el aprendizaje y el rendimiento escolar, hasta el punto de generar confusiones derivadas de la superposición de estos conceptos (Bravo, 2012; Heaven \& Ciarrochi, 2012; Strobel, Behnke, Gärtner \& Strobel, 2019); además del posicionamiento de visiones que atribuyen a la inteligencia un rol central o bien predictivo del rendimiento escolar (Jensen, 1973; Bravo, Villalón \& Orellana, 2004; Strobel, Behnke, Gärtner \& Strobel, 2019).

Por otra parte, en busca de alcanzar conceptualizaciones que integren los elementos que componen el constructo de inteligencia surge la perspectiva factorial (Ferreira, Zanini \& Seabra, 2015; Rosas et al., 2005). Esta perspectiva se centra en indagar la estructura misma de la inteligencia a través de exigentes métodos estadísticos. Desde esta perspectiva surgen diferentes 
enfoques factoriales, desde aquellos que postulan la presencia de un factor principal que explicaría la estructura de este constructo a otros que postulan visiones multifactoriales de este concepto (Arancibia, Herrera \& Strasser, 2004).

La concepción de inteligencia cristalizada (Gc) e inteligencia fluida (Gf) constituye otra perspectiva teórica relevante en la conceptualización del constructo inteligencia, donde convergen elementos derivados de la perspectiva factorial y psicométrica (Arán Filippetti, Krumm, \& Raimondi, 2015; Pérez \& Medrano, 2013). La inteligencia cristalizada (Gc) reflejaría el conocimiento adquirido por medio de procesos culturales y educativos, por lo cual se encontraría más relacionada con el nivel de escolarización y aprendizaje; mientras que la inteligencia fluida (Gf) representaría aspectos menos adquiridos y más relacionados con la capacidad abstracta en la resolución de problemas (Arán Filippetti et al., 2015, Ardila, 2011; Cattell, 1943).

Como complemento a la visión tradicional de inteligencia que enfatiza prioritariamente elementos de tipo cognitivos y abstractos, hemos sido testigo en las últimas décadas del surgir de nuevas concepciones de inteligencia que acentúan elementos relativos a la emocionalidad, a aspectos sociales, a elementos artísticos e incluso a cualidades de tipo intrapersonal (CobosSánchez, Flujas-Contreras \& Gómez-Becerra, 2017; Goleman, 2000; Isaza \& Calle, 2016). Este cambio de paradigma responde a la necesidad de ampliar el constructo de inteligencia a otras dimensiones más cercanas a la cotidianidad de la vida de las personas (Goleman, 2000; Mesa, 2018; Rosas et al., 2005).

También encontramos perspectivas de estudio más contemporáneas que se enfocan en los procesos capaces de regular no sólo la actividad cognitiva, sino también aquellas conductuales, sociales y emocionales. Nos referimos específicamente a las funciones ejecutivas (FE), denominadas también habilidades cognitivas de alto orden que contemplan tanto aspectos cognitivos (FE cool) como emocionales y motivacionales (FE hot) (Zelazo \& Muller, 2002) y subyacen a la conducta inteligente. Si bien existe diferencia entre los constructos relativos a la inteligencia y a las FE, podemos también retener que ambos son componentes centrales de la cognición y resultan relevantes no sólo en la adaptación cognitiva, sino también social, conductual y afectiva (García-Molina, Tirapu-Ustárroz, Luna-Lario, Ibáñez \& Duque, 2010). No obstante, los estudios que han examinado la relación entre la inteligencia, valorada desde pruebas psicométricas y las FE, han arrojado resultados contradictorios. En efecto, en poblaciones de niños y adolescentes se han informado desde muy bajas correlaciones (Arán Filippetti et al., 2015; Ardila, Pineda \& Rosselli, 2000; Montoya-Arenas, Trujillo-Orrego y Pineda-Salazar, 2010; Welsh, Pennington \& Groisser, 1991), hasta correlaciones significativas entre las diferentes FE y la inteligencia cristalizada (Brydges, Reid, Fox, \& Anderson, 2012), fluida (Brydges et al., 2012; Duan, Wei, Wang \& Shi, 2010) y general (Arffa, 2007). Esto ha llevado a concluir que estos constructos se superponen solo en algunos aspectos y que, si bien tanto las FE como la inteligencia hacen referencia a la capacidad del sujeto para adaptarse a su entorno, no pueden emplearse como términos intercambiables (García-Molina et al., 2010). Además, de relevar que los test de inteligencia no utilizan en la operacionalización del constructo elementos relativos a los procesos ejecutivos, aspecto significativo y congruente con el enfoque que postula que las FE constituirían una esfera relevante de la cognición pero relativamente independiente del CI (Arán Filippetti et al., 2015; Montoya-Arenas et al., 2010; Welsh et al., 1991).

\section{Funciones ejecutivas (FE).}

Las FE se configuran como un conjunto de procesos cognitivos que se vinculan directamente con la capacidad de autorregulación, la intencionalidad y la toma de decisiones (Arán Filippetti \& López, 2013; Goldberg, 2001; Miyake \& Friedman, 2012). Es decir, constituyen un conjunto de procesos de control capaces de regular el pensamiento y la conducta de las personas, en la toma de decisiones y en el despliegue de sus potencialidades (Miyake \& Friedman, 2012). 
Existen evidencias que sostienen que el desarrollo de las FE, si bien no se delimita exclusivamente a la infancia, es en esta etapa donde presentaría una mayor intensidad y la posibilidad de instauración de competencias metacognitivas relevantes para el desarrollo y adaptación integral de niños y niñas (Arán Filippetti \& López, 2013; Best, Miller \& Naglieri, 2011; Flores, Castillo \& Jiménez, 2014; Pennequin, Sorel \& Fontaine, 2010; Stelzer, Cervigni \& Martino, 2011). Este desarrollo, se encuentra estrechamente relacionado y supeditado a elementos de tipo neurobiológicos y ambientales (Gaitán \& Rey, 2013). En efecto, el sustrato neurológico de las FE lo encontramos en la corteza prefrontal y la corteza cingulada, en conexión con áreas corticales y subcorticales (Heyder, Suchan \& Daum, 2004; Stelzer et al., 2011). El factor ambiental, también resulta relevante para el desarrollo de las FE, pues estas estructuras serían susceptibles no sólo de ser modificadas por el contexto socioambiental circundante, sino también estimuladas y sostenidas por él (Noble, Norman \& Farah, 2005). En efecto, la conformación de las redes neuronales centrales para el despliegue y articulación de las FE, se encuentra estrechamente relacionada con la calidad de la estimulación ambiental (Hackman \& Farah, 2008; Noble et al., 2005; Zelazo, 2003).

Entre las FE más relevantes descriptas en la literatura, se destacan el control inhibitorio de respuestas impulsivas, la capacidad de flexibilidad cognitiva que permite cambiar estrategias de acción cuando se considera necesario y oportuno, la memoria de trabajo y la capacidad de planificación y organización (Anderson, 2002; Arán Filippetti \& López, 2013; Davidson, Amso, Anderson \& Diamond, 2006; Miyake \& Friedman, 2012; Sastre-Riba, 2006). Dado que estos procesos cognitivos de alto orden se manifiestan en la autorregulación de la actividad cognitiva, conductual y emocional, la inserción de su estudio al campo educativo ha sido particularmente valiosa. Estudios en esta línea han demostrado que las FE predicen el desarrollo de habilidades pre-académicas (Espy, McDiarmid, Cwik, Stalets, Hamby, \& Senn, 2004; Shaul \& Schwartz, 2014) así como el aprendizaje y el rendimiento académico en edad escolar (Jacobson Williford, \& Pianta, 2011; St Clair-Thompson \& Gathercole; 2006; Thorell, Veleiro, Siu, \& Mohammadi, 2013). Además, se reconocen como procesos cruciales para la autonomía del niño en su funcionamiento diario (Rosenberg, 2014), el desarrollo de competencias socioemocionales (Riggs, Jahromi, Razza, Dillworth-Bart, \& Mueller, 2006), la comprensión emocional (Martins, Osório, Veríssimo, \& Martins, 2016) y el potencial creativo en edad escolar (Krumm, Arán Filippetti \& Gutiérrez, 2018). Tanto las FE como las habilidades metacognitivas contribuyen diferencialmente al rendimiento académico (Bryce, Whitebread, \& Szücs, 2015) y serían un predictor del éxito escolar aún más importante que el nivel de inteligencia general (Arán Filippetti \& Richaud, 2017).

Teniendo en cuenta las implicancias de reconocer estos procesos cognitivos como habilidades atribuibles a un comportamiento inteligente, centrales para alcanzar un aprendizaje autorregulado, los objetivos del presente estudio fueron (a) indagar sobre las concepciones que niñas y niños de 8 a 12 años de edad otorgan al concepto de inteligencia, (b) examinar las características que le atribuyen al concepto y (c) conocer la valoración que hacen de las propias capacidades intelectuales. Para esto, se utilizó una metodología de tipo cualitativa, específicamente, se trabajó desde la Teoría Fundamentada, perspectiva metodológica que permite descubrir los conceptos que se desprenden del contexto investigado y que subyacen al concepto de estudio (Charmaz, 2007), por lo tanto, nos entregan conocimiento situado.

\section{Método}

Se utilizó una metodología de tipo cualitativa, ya que este enfoque resulta coherente con la finalidad de recoger información respecto de las concepciones que niñas y niños otorgan al constructo de inteligencia, como lo caracterizan y la autopercepción del propio desempeño intelectual. Para llevar a cabo este objetivo, se trabajó desde la perspectiva de la teoría fundamentada, en cuanto este enfoque nos permite identificar los determinantes del constructo de inteligencia que manejan niños y niñas. Es decir, a través de este método, se llevó a cabo un acercamiento a los significados proporcionados por parte de los participantes a esta investigación 
(Ruíz, 2003), posibilitando la construcción de una teoría situada del constructo en estudio, a través de la interrelación de las categorías y subcategorías emanadas del contexto investigado (San Martín, 2014; Vivar, Arantzamendi, López-Dicastillo \& Gordo, 2010).

\section{Participantes.}

Se utilizó un muestreo de tipo teórico, por lo cual la selección de participantes se llevó a cabo según la necesidad de construcción de una teoría explicativa del constructo de inteligencia, a partir de las categorías y subcategorías emanadas de las percepciones y caracterizaciones que la muestra de estudio otorgó al constructo de inteligencia. El proceso de recolección de datos se llevó a cabo hasta el criterio de saturación teórica, proceso que culmina "cuando la recogida de nuevos datos ya no aporta información adicional o relevante para explicar las categorías existentes o descubrir nuevas categorías" (Vivar, et al., 2010, p. 287). La muestra quedó constituida por 16 sujetos de edades comprendidas entre 8 y 12 años. La elección de los niños y niñas responde a un criterio de tipo cronológico y a la exigencia de ser alumnos regulares del sistema educacional chileno. La selección fue de tipo intencional, respondiendo a la voluntad de los participantes y a la autorización de los padres por ser menores de edad (França-Tarragó, 2008; Losada \& López, 2003; Ruíz, 2003).

\section{Técnicas de producción de datos.}

Para poder cumplir con el objetivo de conocer los significados que los niños y niñas le atribuían al concepto de inteligencia, se utilizó la técnica de entrevistas estructuradas, con el fin de identificar la conceptualización de inteligencia, las características que le atribuyen y la valoración que hacen los participantes de este estudio respecto de sus propias habilidades intelectuales. La entrevista estructurada se caracteriza porque las preguntas que la componen son preestablecidas con anticipación y se plantean en el mismo orden a los participantes en estudio (Fernández, 2001). Los tópicos tratados en las entrevistas ${ }^{1}$ respondían específicamente a tres elementos: concepción, características y autopercepción de inteligencia manejados por los destinatarios de este estudio.

\section{Procedimiento.}

En primer lugar, se procedió a contactar a niñas y niños que cumplieran con las condiciones requeridas por el estudio a través de sus padres o tutores legales, luego se solicitó el consentimiento informado por parte de éstos y antes de iniciar las actividades se les consultó a los mismos niños y niñas su disposición a participar de las entrevistas (asentimiento), informando la posibilidad de abandonar las actividades en el momento que lo estimaran pertinente (FrançaTarragó, 2008). Una vez que los niños y niñas respondieron a la encuesta, se procedió a transcribirlas integralmente con la finalidad de llevar a cabo un análisis detallado de ellas. El análisis de los datos se llevó a cabo a través de la teoría fundamentada, utilizando el programa ATLAS.ti versión 8. Con estos hallazgos se construyó un modelo en donde se definen las relaciones entre las categorías y subcategorías.

\section{Análisis de los datos.}

El análisis de los datos se llevó a cabo siguiendo la perspectiva de la teoría fundamentada, caracterizada por un enfoque sistemático inductivo y comparativo para analizar los datos y levantar la construcción de teorías de los tópicos estudiados (Charmaz, 2007; Charmaz, 2014). Para tales efectos, se procedió a transcribir y analizar rigurosamente las entrevistas, con el fin de identificar unidades de significado y asignar códigos iniciales que representasen la acción o idea manifiesta por los participantes en el estudio (Gaete, 2014). La codificación constituye un proceso relevante para la metodología propuesta, razón por la cual se procedió en un primer momento a ejecutar una codificación abierta, donde se identificaron y recogieron temas, elementos,

\footnotetext{
${ }^{1}$ El guión de la entrevista se encuentra en anexos.
} 
características y/o patrones (Bonilla \& López, 2016; Gaete, 2014; Reyes, Altamar, Aguirre \& Murillo, 2014). Una vez concluida la codificación abierta, se procedió a identificar las categorías centrales, a través de un proceso de análisis comparativo de los códigos encontrados. A este proceso de contraste se le denomina codificación axial (Vivar et al., 2010). Luego, a través del proceso de codificación selectiva se sintetizan e integran los datos del estudio, descubriendo la centralidad de algunas categorías de análisis, sea por su capacidad explicativa como por el sentido que otorgan a las relaciones y a toda la información encontrada, dando como resultado la teoría de inteligencia manejada por los niños y niñas que participaron de este estudio, fundamentada en sus respuestas (Reyes et al., 2014; Vivar et al., 2010)

\section{Resultados}

\section{Significado atribuido al concepto inteligencia .}

Los resultados respecto del significado atribuido al constructo de inteligencia, producto de los análisis de las relaciones entre las categorías y subcategorías, revelan seis conceptos claves a la hora de significar el término. El concepto relativo al manejo o posesión de contenido escolar se desprende como central a la hora de precisar el constructo de inteligencia; de hecho los sujetos participantes en el estudio definen inteligencia, principalmente, en base a la capacidad de manejar contenido impartido en contexto escolar, sea a nivel general, como específico en el ámbito de las matemáticas y lenguaje principalmente ("la inteligencia para mi es saber todas las materias, principalmente ser muy bueno para las matemáticas y lenguaje", "yo creo que inteligencia es saberlo todo, digo todo y de todas las asignaturas", "es saber de todos los ramos, matemáticas, lenguaje y todas las asignaturas, pero sin olvidarse", "yo pienso que la inteligencia es saber todo lo que nos enseñan"). Otro concepto, relacionado con el anterior, aunque con menor fuerza de aparición, lo constituye el rendimiento escolar, referido específicamente al éxito cuantitativo que se manifiesta en las calificaciones escolares y que en palabras de los participantes se expresa en: "inteligencia es sacarse buenas notas, es tener puros sietes" ", "es sacarse sietes en todo, creo que es tener las mejores notas del curso" (ver figura 1).

Resulta relevante también, que, a la hora de definir inteligencia, aparezcan con intensidad considerable, aseveraciones relativas a procesos reconducibles a las funciones ejecutivas (FE), tales como procesos autorregulatorios y componentes ejecutivos relativos al control inhibitorio, memoria de trabajo, fluidez verbal y capacidad de planificación y organización. Los procesos autorregulatorios, constituyen las categorías que se presentan con mayor fuerza, en las respuestas de niños y niñas para significar el constructo de inteligencia. Aseveraciones tales como "saber lo que uno procesa o estudia para una prueba o algo así", "ocupar el ciento por ciento de tu cerebro, o de tu cabeza", "usar información que te han enseñado en el pasado y ocuparla" constituyen elementos proporcionados por parte de los participantes reconducibles a la subcategoría de regulación cognitiva. Así también, aparecen expresiones relativas a la capacidad de regular la propia conducta ("ser inteligente es comportarse bien y escuchar", "obedecer y hacer las tareas y pararse sólo cuando es necesario, no es pararse y molestar a los compañeros a cada rato") y a la habilidad de resolver problemas ("es poder resolver ejercicios dificiles y no dejarse vencer", "ser inteligente es como resolver problemas y no quedarse con las dudas") como significaciones relevantes para el constructo en estudio. Resulta relevante observar que estos procesos autorregulatorios son factibles gracias a la actuación de diferentes componentes ejecutivos, tales como el control inhibitorio, la memoria de trabajo, la fluidez verbal, flexibilidad cognitiva, la capacidad de panificación entre otras funciones.

La capacidad de inhibir respuestas impulsivas y automatizadas, mientras se persigue un objetivo, constituye otra de las categorías presente en las respuestas de los informantes a la hora de conceptualizar el constructo de inteligencia. Esta función requiere de la capacidad de aprender a ignorar estímulos distractores o no relevantes y focalizarse en aquellos reconducibles a un fin

\footnotetext{
${ }^{2}$ Nota máxima contemplada en el sistema escolar chileno.
} 
específico (Gaitán \& Rey, 2013). Expresiones tales como "inteligencia es estudiar mucho y no distraerse con otras cosas", "es esforzarse para entender y dejar de pensar en otras cosas como el fútbol, o el play", "es poner atención en clases, hacer tareas y tratar de no conversar tanto con las amigas", constituyen elementos que dan cuenta del control inhibitorio como aspecto presente en la significación de inteligencia, que realizan niñas y niños.

En la figura 1, también podemos observar conceptualizaciones de inteligencia orientadas al despliegue de habilidades sociales ("la inteligencia para mi es ser buena persona, llevarme bien con los demás", "es saber tener amigos y sentirse bien con la familia") y con menor intensidad a aspectos de regulación externa, referidos específicamente al reconocimiento de terceras personas de las capacidades o destrezas individuales ("es que me feliciten, mi padres y profesores).

La categoría de incertidumbre presente a la hora de solicitar significaciones del constructo de inteligencia constituye un elemento de notable interés en el análisis efectuado. En efecto, podemos observar que esta categoría aparece en tercer lugar, si consideramos la jerarquía o peso de aparición y manifiesta desazón, ansiedad y cierta perplejidad al sentirse enfrentados a este tópico de estudio ("bueno para mí la inteligencia es como... las personas que se dedican a...", "es algo que... con lo que nosotros...", "no sé cómo decirlo... esta pregunta es fácil, pero me pone nerviosa..."

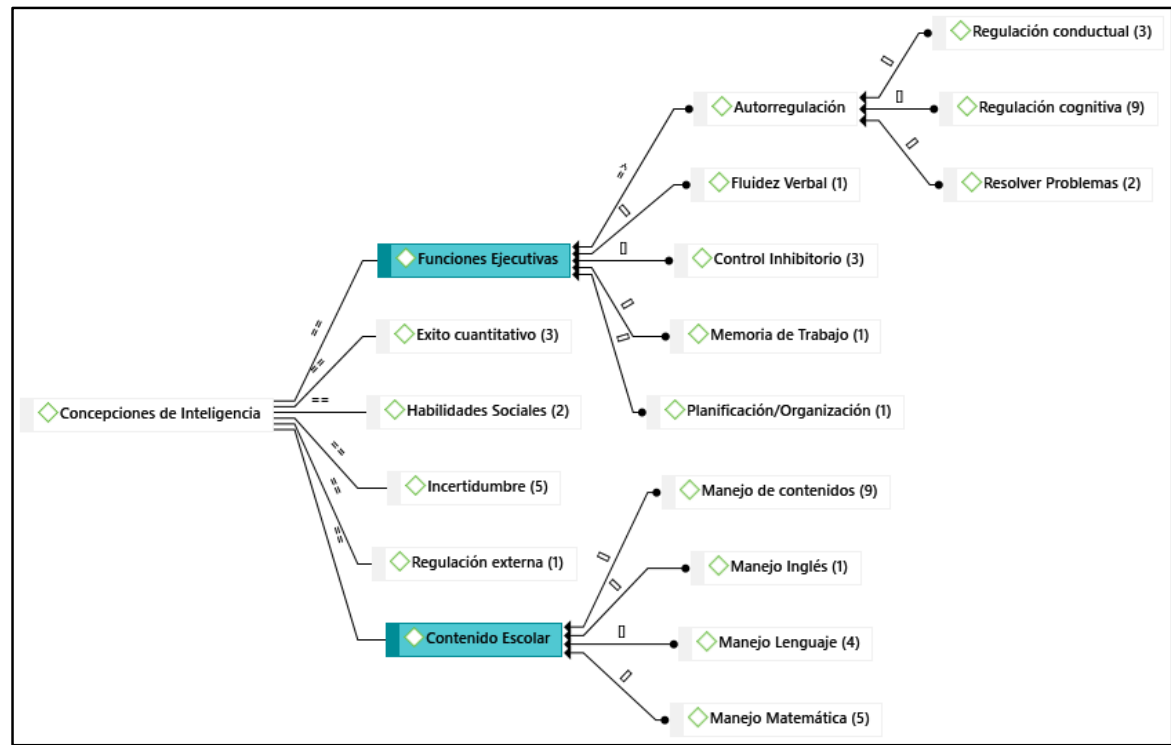

Figura 1. Categorías y relaciones de las conceptualizaciones del constructo inteligencia

\section{Características atribuidas a una niña o niño considerado inteligente.}

Las características atribuidas a una niña o niño considerado inteligente, constituye el segundo tópico de análisis. Los participantes, como se visualiza en la figura 2, atribuyen principalmente características relativas al éxito cuantitativo y al manejo o adquisición de FE. El aspecto relativo al éxito cuantitativo se refiere específicamente al rendimiento escolar, es decir un niño o niña inteligente, según las opiniones de sus compañeros, debiera obtener buenas calificaciones, lo que queda de manifiesto en las siguientes expresiones: "se saca buenas notas", "se saca puros sietes", no tiene notas rojas", "siempre se saca buenas notas". Resulta interesante notar que el manejo de contenidos, si bien permanece como cualidad atribuida a un sujeto retenido inteligente, su intensidad de aparición se reduce con respecto a la relevancia otorgada en la significación o conceptualización que los participantes hacen del constructo de inteligencia.

Las FE constituyen los elementos que emergen con mayor intensidad en las respuestas otorgadas por los participantes a la hora de caracterizar sujetos retenidos inteligentes. Entre ellas 
destacan: la autorregulación, el control inhibitorio, la capacidad de organización y la fluidez verbal. Como en el caso de las conceptualizaciones de inteligencia, la caracterización que hacen los mismos niños y niñas del constructo antes mencionado, se orienta también a elementos reconducibles a funciones autorregulatorias, tales como regulación cognitiva ("pone atención en clases", "tiene pensamientos claros"), conductual ("se porta bien en la sala", "tiene que mantener una disciplina en la sala de clases", "la inteligencia también viene con buen comportamiento"), emocional ("no tienen problemas emocionales porque si tiene inteligencia sabrá cómo controlarse") y perseverancia ("no se rinde fácil", "si le va mal en una tarea vuelve a intentar siempre"). Llama la atención que si bien priman elementos relacionados con la autorregulación cognitiva (manifestación de las FE cool), como en el caso de la conceptualización del constructo de inteligencia, emergen con mayor intensidad aspectos relativos a la regulación conductual y emocional (manifestación de las FE hot) al momento de caracterizar un sujeto retenido inteligente.

Otras de las habilidades ejecutivas atribuidas a la inteligencia se relaciona explícitamente con el control inhibitorio, aspecto que adquiere mayor fuerza en la caracterización que los sujetos hacen del constructo en estudio y que se traduce en expresiones tales como: "se concentra, no se preocupa de lo que están haciendo los demás", "primero que todo tiene que esforzarse en lo que hace y después puede hacer lo que quiera", "es un niño responsable, que primero hace sus tareas y después juega con los amigos". Aseveraciones que aluden a la capacidad de dominar respuestas más instintivas y automáticas para dar paso a fomas de procesamiento más analítico y reflexivo en vista de la consecución de objetivos específicos, que a la vez posibiliten modalidades más adaptativas (Flores et al., 2014; Miyake, Friedman, Emerson, Witzki, Howerter \& Wager, 2000).

Dentro de las FE también aparece la organización escolar, entendida como la capacidad de ordenar, priorizar y secuenciar información o estrategias en vistas al perseguimiento de objetivos escolares (Gioia, Isquith, Guy \& Kenworthy, 2017) y que en palabras de las niñas y niños participantes en el estudio se expresa en aseveraciones como: "siempre toma apuntes", "hace todas las tareas", "tiene hábitos de estudio", "siempre trae sus materiales". Respuestas que nos permiten discriminar no sólo las principales estrategias utilizadas para alcanzar objetivos escolares propuestos, sino también la frecuencia de éstas. La componente ejecutiva de fluidez verbal constituye otra de las subcategorías que emerge, aunque con menor peso, al momento de caracterizar el constructo de inteligencia y se expresa en el discurso de los participantes como: "sabe hablar bien... mejor que los demás", "sabe leer rápido y hablar bien"; expresiones que enfatizan la capacidad de organizar y transmitir información de modo adecuado y con cierta velocidad (Benjumea, Ocampo, Vega, Hernández \& Tamayo, 2016).

En la figura 2, también podemos observar la presencia de otras dos categorías que emergen a la hora de caracterizar el constructo en estudio. La primera de ellas se refiere al desarrollo de habilidades sociales, entendidas específicamente como la capacidad de establecer relaciones cordiales y solidarias con los demás ("yo creo que un niño inteligente no es peleador y sabe hacerse amigos", "inteligente es quien no pelea y comparte sus materiales", "es buena persona en su vida cotidiana y generoso"). Mientras que, la segunda, se refiere al reconocimiento externo y aunque aparece con menor intensidad resulta interesante, pues en este caso la caracterización viene dada por la entrega de un objeto externo que acredite la posesión de la habilidad en estudio ( "siempre se gana diplomas"). 


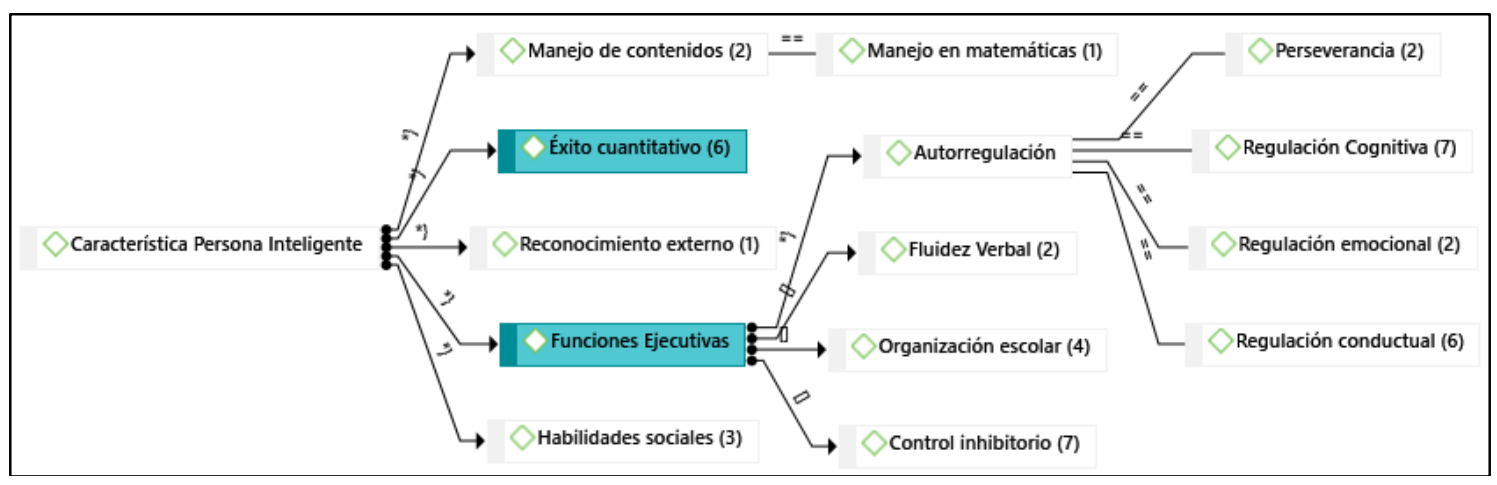

Figura 2. Categorías referentes a la caracterización de un sujeto inteligente.

\section{Consideración sobre la propia inteligencia.}

En relación con la tercera y última temática de estudio, referida a la interrogante respecto de la consideración de la propia inteligencia, los resultados denotan que tan sólo un $56 \%$ de los participantes se retienen niñas o niños inteligentes (ver figura 3). Entre las argumentaciones que sustentan dicha consideración prima el hecho de obtener buenas calificaciones, en efecto el éxito cuantitativo constituye el argumento principal de la valoración intelectual positiva y se expresa en aseveraciones tales como: "siempre me he sacado buenas notas y me va bien en el colegio", "porque he mejorado mis notas, tengo buen promedio", "me va bien en las pruebas, tengo buenas notas", "mi promedio de notas es siempre el mejor del curso". En concordancia con este sustento, emerge la categoría relativa al manejo de contenidos escolares, como argumento de la consideración intelectual positiva, aunque con un índice mucho menor de aparición y se expresa explícitamente en aseveraciones que afirman: "me va súper bien en matemáticas, también en lenguaje y en todas las asignaturas".

La utilización de recursos referidos a la regulación cognitiva ("he colocado atención siempre en las clases, por eso puedo contestar bien las preguntas que me hacen", "porque aprendo rápido"), al control inhibitorio ("cuando me lo propongo, pongo atención y dejo de conversar") y a la perseverancia ( "yo nunca me rindo con ninguna tarea") constituyen elementos relacionados con las FE que también aparecen para apoyar y fundamentar una valoración positiva de las propias habilidades intelectuales.

Por otra parte, resulta relevante que un $46 \%$ de niñas y niños participantes en el estudio manifiesten una consideración intelectual negativa de sí mismos. Entre las argumentaciones centrales que sostienen esta valoración, encontramos la percepción de déficit en la autorregulación cognitiva, conductual y en el despliegue de FE relacionadas con el control inhibitorio. En efecto, dentro de los fundamentos relacionados con la regulación cognitiva encontramos las siguientes aseveraciones: "igual yo me esfuerzo por hacer las tareas y estudiar, pero me va mal, así como inteligente inteligente no soy", "a veces estudio mucho y cuando llego a la prueba no, no se me ocurre nada", "nunca respondo bien a una pregunta". Argumentaciones que ponen de manifiesto un cierto grado de dificultad para gestionar y manejar los procesos cognitivos propios. Del mismo modo, encontramos afirmaciones que explicitan dificultades en la regulación de los propios impulsos ("en realidad no me esfuerzo mucho, quiero pero no lo hago", "algunas veces soy flojo y no me gusta estudiar, prefiero hacer otras cosas", "los estudios los he dejado un poco al lado, prefiero jugar") y en el control de la conducta ("no tengo muy buen comportamiento, no obedezco", "me porto mal en clases, siempre me estoy moviendo y a veces soy peleador"). De igual modo, resulta curioso que a la hora de sostener una consideración negativa de las propias capacidades intelectuales, los participantes en el estudio recurran con menor intensidad a argumentos relativos al rendimiento escolar ("Me saco malas notas", "tengo muy malas notas") y privilegien elementos relacionados con los componentes ejecutivos. 


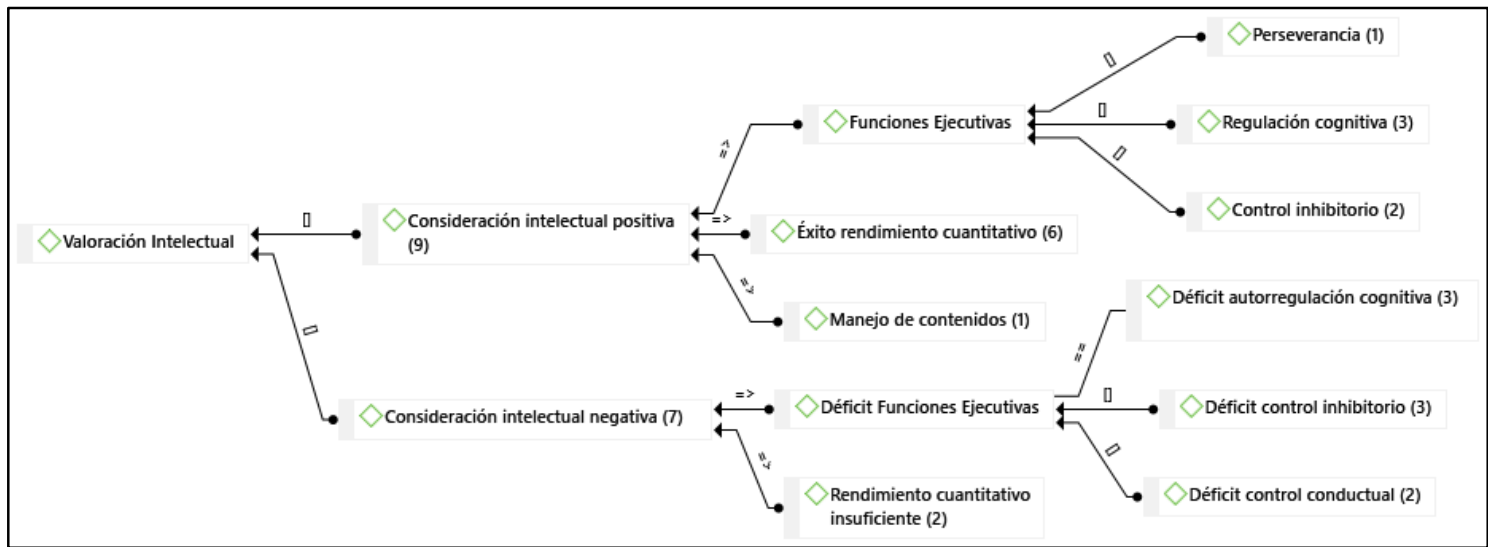

Figura 3. Categorías y relaciones referidas a la propia valorización intelectual

\section{Discusión}

El objetivo de este estudio fue conocer las concepciones que niñas y niños, de edades comprendidas entre 8 y 12 años, otorgan al concepto de inteligencia, las características que le atribuyen y la valoración de las propias capacidades intelectuales. Específicamente se buscó descubrir, a través de la teoría fundamentada, los modelos explicativos relacionados con la teoría de inteligencia que manejan niñas y niños escolarizados en nuestros contextos, aspecto relevante pues los resultados nos develan información situada, es decir conceptos, conjeturas y proposiciones que emergen de los informantes y no exclusivamente de supuestos a priori (Charmaz, 2014; San Martín, 2014, Vivar et al., 2010), por lo cual resulta altamente probable que estas significaciones y atribuciones influyan significativamente en la experiencia escolar de muchos de ellos.

En primer lugar, los resultados manifiestan que el manejo de contenidos escolares constituye la categoría principal a la hora de conceptualizar el contructo de inteligencia. Información que, junto a elementos referidos a un rendimiento escolar exitoso, conforman parte significativa de la teoría situada de las concepciones de inteligencia que manejan los sujetos participantes de este estudio. Significaciones referidas al contenido y al rendimiento escolar que encuentran una fuerte concordancia con enfoques teóricos que entienden la inteligencia no sólo como procesos abstractos y lógicos (Gf), sino también cristalizada en conocimiento y contenidos alcanzados explícitamente a través de la experiencia educativa y cultural $(\mathrm{Gc})$ (Arán Filippetti et al., 2015, Ardila, 2010; Cattell, 1943). El énfasis otorgado a los contenidos escolares al momento de significar la inteligencia también ha venido favorecido por el desarrollo de la perspectiva psicométrica, la que en vista de la dificultad experimentada para medir inteligencia de foma directa, la ha evaluado mediándola a través de contenidos escolares, utilizado muestras también provenientes de estos contextos (Isaza \& Calle, 2016; Rosas, et al., 2005). Desde esta base, resulta sencillo entender la asociación que se ha instaurado no sólo entre los conceptos de inteligencia y aprendizaje, sino también entre estos términos y el rendimiento escolar (Bravo, 2012; Heaven \& Ciarrochi, 2012), significaciones del constructo inteligencia, que emergen también hoy en las opiniones de niñas y niños que se educan en nuestros contextos educativos. Correspondencia intensa entre conocimiento situado y aquel teórico ya sistematizado, que no se evidencia en el caso de significaciones relativas a aspectos sociales, los cuales aparecen con intensidad mucho menor en el discurso de los participantes.

Por otra parte, observamos con admiración el emerger de contenidos relativos a las FE como parte relevante de la teoría situada, que manejan niñas y niños, del constructo inteligencia. A este respecto advertimos, que las significaciones de inteligencia se concentran principalmente no en los componentes ejecutivos específicos, sino en la capacidad de autorregulación que estas 
funciones posibilitan. A este punto cobra sentido reflexionar sobre las implicancias del concepto de FE, el cual se focaliza en destacar la multidimensionalidad de este constructo, para enfatizar que comprende un conjunto de funciones cognitivas, que posibilitan o hacen factible la capacidad de autorregulación no sólo cognitiva, sino también conductual, afectiva y social, consintiendo la toma de decisiones y el despliegue de potencialidades (Arán Filippetti et al., 2013; Gioia et al., 2017; Goldberg, 2001; Miyake \& Friedman, 2012).

La caracterización atribuida a un sujeto retenido inteligente constituye el segundo tópico de análisis. Los resultados posicionan a las FE como las categorías que priman a la hora de caracterizar el constructo en estudio, en efecto, emergen aseveraciones relacionadas con procesos autorregulatorios, factibles por la presencia de componentes ejecutivos relativos al control inhibitorio, la capacidad de organización y la fluidez verbal. Dentro de la categoría de autorregulación, emergen elementos relativos a la regulación cognitiva y a la regulación conductual. La regulación cognitiva, constituye la dimensión que emerge con mayor intensidad, no sólo al momento de caracterizar el desempeño inteligente, sino también al conceptualizar el constructo en estudio y comprende elementos referidos al control intencional del pensamiento, a procesos metacognitivos, a la utilización de recursos intelectuales disponibles, a la actualización de potencialidades y a la transferencia de la información. Mientras que la regulación conductual, expresa los esfuerzos de control deliberado por adecuar la conducta a objetivos escolares, tales como seguir instrucciones, ocupar una posición específica en el aula y silenciarse para atender a estímulos relevantes de la experiencia escolar; si bien esta subcategoría constituye una temática constante en el estudio, adquiere mayor fuerza al momento de caracterizar un sujeto retenido inteligente. Lo mismo acontece con el componente ejecutivo del control inhibitorio, aspecto que emerge de las respuestas de los sujetos participantes en el estudio, como característica relevante del desempeño inteligente y alude a la capacidad de ignorar estímulos distractores o poco relevantes (Gaitán \& Rey, 2013) y se expresa en el control deliberado de los procesos de concentración, donde la atención sostenida, selectiva y monitoreada adquiere un rol importante.

Entre las características atribuídas a un individuo considerado inteligente, emergen también elementos referidos a la posesión de contenidos escolares y al rendimiento escolar exitoso, categorías ya instaladas en el primer tópico de análisis. Aunque resulta necesario advertir que la categoría referida al rendimiento escolar exitoso adquiere mayor fuerza de aparición, respecto del manejo de contenidos al momento de caracterizar el desempeño inteligente. Aspecto relevante, pues si bien el contenido escolar constituye un aspecto importante de la conceptualización de inteligencia, su concreción viene estimada a partir del rendimiento escolar, específicamente en el ámbito de las calificaciones. Estos resultados nos evidencian vestigios de las transposiciones y confusiones que perduran hasta el día de hoy respecto de la conceptualización de inteligencia y es producto en gran medida de los mecanismos utilizados para poder medir este constructo.

Con respecto al tercer tópico de análisis, cuyo objetivo se orientaba a conocer la valoración que hacen los participantes de las propias capacidades intelectuales, resulta al menos interesante observar el surgir nuevamente de las categorías relativas al rendimiento escolar exitoso y al funcionamiento ejecutivo, como fundamentos centrales de la propia valoración intelectual; aunque se evidencian distintos niveles de intensidad en la saturación de estas categorías, niveles que dependen del tipo de consideración que se tenga del propio intelecto. En efecto, las niñas y niños que revelan una valoración positiva de su capacidad intelectual fundamentan su consideración en las categorías antes señaladas, con niveles de aparición bastante similares. Mientras, que los sujetos que presentan una valoración negativa de sus recursos intelectuales sostienen su consideración principalmente en elementos referidos a dificultades en las FE, especialmente a déficits en los procesos inhibitorios, de control cognitivo y conductual.

A este punto, resulta importante destacar que los elementos relativos a la regulación cognitiva, regulación conductual (adscritos a la categoría de autorregulación) y control inhibitorio constituyen aspectos relevantes emanados del contexto investigado, no sólo al momento de 
valorar la propia capacidad intelectual, sino también al conceptualizar y caracterizar el constructo de inteligencia. Al analizar estas funciones podemos percatarnos de elementos diferenciadores entre ellos, tales como la acentuación de recursos cognitivos-intelectuales, la intencionalidad en el control de la conducta y de los procesos que sostienen la concentración. No obstante, encontramos elementos transversales que los unen, como la capacidad de inhibir o contener impulsos, tendencias, ideas, intereses y respuestas automatizadas, en vista de regular la cogniciónintelecto, la conducta y la atención-concentración; aspectos todos, relacionados y conectados con habilidades de autocontrol o autorregulación en la toma de decisiones, centrales para alcanzar un aprendizaje autorregulado. Es importante enfatizar entonces que, si bien las FE emergen con intensidad en los modelos explicativos de los participantes, no se configura como sinónimo del constructo inteligencia, sino como habilidades cognitivas diferenciadas (aunque no necesariamente relacionadas) ligadas al concepto de inteligencia.

Los resultados de este estudio adquieren gran relevancia no sólo para el ámbito educativo, sino también para la psicología educacional y neuroeducación, en cuanto nos entrega información situada respecto de la teoría de inteligencia que manejan niñas y niños de nuestros contextos educativos, constituyendo el modelo explicativo por excelencia que utilizan al momento de significar sus vivencias y procesos relacionados no sólo con el aprendizaje escolar, sino también con elementos motivaciones, afectivos y sociales relevantes para el despliegue de potencialidades y el desarrollo integral. Analizar las concepciones, características y valoraciones de la inteligencia, constituyó una estrategia para acercarnos a las significados, creencias y atribuciones presentes en población infantil y aunque reconocermos límites relativos a la generalizacion de los resultados, elementos asumidos en las opciones metodológicas, también somos conscientes de la riqueza de contar con una teoría situada del constructo estudiado, que nos entregue información valiosa para comprender y emprender nuevas perspectivas de análisis.

\section{Referencias}

Anderson, P. (2002). Assessment and Development of Executive Function (EF) During Childhood. Child Neuropsychology, 8(2), 71-82. Doi: https://doi.org/10.1076/chin.8.2.71.8724

Arán Filippetti, V., \& López, M. B. (2013). Las funciones ejecutivas en la clínica neuropsicológica infantil. Psicología desde el Caribe, 30(2), 380-415. Recuperado de: http://www.redalyc.org/comocitar.oa?id=21328601008

Arán Filippetti, V. A., Krumm, G., \& Raimondi, W. (2015). Funciones Ejecutivas y sus correlatos con Inteligencia Cristalizada y Fluida: Un estudio en Niños y Adolescentes. Neuropsicología Latinoamericana, 7(2), 24-33. Recuperado de: http://neuropsicolatina.org/index.php/Neuropsicologia_Latinoamericana/article/view/21 3

Arán Filippetti, V., \& Richaud, M. C. (2017). A structural equation modeling of executive functions, IQ and mathematical skills in primary students: Differential effects on number production, mental calculus and arithmetical problems. Child Neuropsychology, 23, 864888. Doi: https://doi.org/10.1080/09297049.2016.1199665

Arancibia, V., Herrera, P., \& Strasser, K. (2004). Manual de psicología educacional. Santiago: Ediciones Universidad Católica de Chile.

Ardila, A., Pineda, D., \& Rosselli, M. (2000). Correlation Between Intelligence Test Scores and Executive Function Measures. Archives of Clinical Neuropsychology, 15, 31-36. Doi: https://doi.org/10.1016/S0887-6177(98)00159-0

Ardila, R. (2011). Inteligencia. ¿Qué sabemos y qué nos falta por investigar? Revista de la Academia Colombiana de Ciencias Exactas, Físicas y Naturales, 35(134), 97-103. Recuperado de: http://www.scielo.org.co/scielo.php?script=sci_arttext\&pid=S037039082011000100009\&lng=en\&tlng=es.

Arffa, S. (2007). The relationship of intelligence to executive function and non-executive function measures in a sample of average, above average, and gifted youth. Archives of Clinical Neuropsychology, 22, 969-978. Doi: https://doi.org/10.1016/j.acn.2007.08.001 
Benjumea, M., Ocampo, E., Vega, J., Hernández, J., \& Tamayo Lopera, D. (2016). Fluidez verbal en estudiantes del grado $11^{\circ}$ de las instituciones educativas Alejandro Vélez Barrientos y José Manuel Restrepo del Municipio de Envigado, según la prueba neuropsicología de las funciones ejecutivas BANFE. Katharsis, 22, 63-85. Recuperado de: http://revistas.iue.edu.co/index.php/katharsis

Best, J. R., Miller, P. H., \& Naglieri, J. A. (2011). Relations between executive function and academic achievement from ages 5 to 17 in a large, representative national sample. Learning and Individual Differences, 21(4), 327-336. Doi: https://doi.org/10.1016/j.lindif.2011.01.007

Bonilla, M., \& López, A. (2016). Ejemplificación del proceso metodológico de la teoría fundamentada. Cinta de Moebio, 57, 305-315. Doi: https://doi.org/10.4067/S0717$554 X 2016000300006$

Bravo, L. (2012). Psicología de las dificultades del aprendizaje escolar. Santiago de Chile: Editorial Universitaria.

Bravo. L., Villalón, M., \& Orellana, E. (2004). Los procesos cognitivos y el aprendizaje de la lectura inicial: diferencias cognitivas entre buenos lectores y lectores deficientes. Estudios Pedagógicos., 30, 7-19. Doi: 10.4067/S0718-07052004000100001

Bryce, D., Whitebread, D., \& Szücs, D. (2015). The relationships among executive functions, metacognitive skills and educational achievement in 5 and 7 year-old children. Metacognition and Learning, 10(2), 181-198. Doi: https://doi.org/10.1007/s11409-0149120-4

Brydges, C. R., Reid, C. L., Fox, A. M., \& Anderson, M. (2012). A unitary executive function predicts intelligence in children. Intelligence, 40, 458-469. Doi: https://doi.org/10.1016/j.intell.2012.05.006

Cabas-Hoyos, K., González-Bracamonte, Y., \& Hoyos-Regino, P. (2017). Teorías de la inteligencia y su aplicación en las organizaciones en el siglo XXI: una revisión. Clío América, 11(22). Doi: http://dx.doi.org/10.21676/23897848.2445

Cattell, R. B. (1943). The measurement of adult intelligence. Psychological Bulletin, 40(3), 153193. Doi: http://dx.doi.org/10.1037/h0059973

Charmaz, K. (2007). Constructing grounded theory. A practical guide through qualitative analysis. Thousand Oaks, CA: Sage.

Charmaz, K. 2014. Grounded theory in global perspective: Reviews by international researchers. Qualitative Inquiry, $20 \quad$ (9), 1074-1084. Doi: https://doi.org/10.1177\%2F1077800414545235

Cobos-Sánchez, L., Flujas-Contreras, J., \& Gómez-Becerra, I. (2017). The role of Emotional Intelligence in Psychological Adjustment among Adolescents. Anales de Psicología, 33(1), 66-73. Doi: http://doi.org/0000-0003-0284-1543

Davidson, M. C., Amso, D., Anderson, L. C., \& Diamond, A. (2006). Development of cognitive control and executive functions from 4 to 13 years: evidence from manipulations of memory, inhibition, and task switching. Neuropsychologia, 44, 2037-2078. Doi: https://doi.org/10.1016/j.neuropsychologia.2006.02.006

Duan, X., Wei, S., Wang, G., \& Shi, J. (2010). The relationship between executive functions and intelligence on 11-to 12-year-old children. Psychological Test and Assessment Modeling, 52, 419-431. Doi: https://doi.org/10.1044\%2F2016_JSLHR-L-15-0310

Espy, K. A., McDiarmid, M. M., Cwik, M. F., Stalets, M. M., Hamby, A., \& Senn, T. E. (2004). The contribution of executive functions to emergent mathematic skills in preschool children. Developmental neuropsychology, 26(1), 465-486. Doi: https://doi.org/10.1207/s15326942dn2601_6

Fernández, R. (2001). La entrevista en la Investigación cualitativa. Revista Pensamiento Actual, 2(3), 14-21. Recuperado de: https://revistas.ucr.ac.cr/index.php/pensamientoactual/article/view/8017/11775

Ferreira, L., Zanini, D., \& Seabra, A. (2015). Executive Functions: Influence of Sex, Age and Its Relationship with Intelligence. Paidéia (Ribeirão Preto), 25(62), 383-391. Doi: https://doi.org/10.1590/1982-43272562201512

França-Tarragó, O. (2008). Ética para psicólogos. Bilbao: Desclée de Brouwer. 
Flores, J. C., Castillo, R. E., \& Jimenez, N. A. (2014). Desarrollo de funciones ejecutivas, de la niñez a la juventud. Anales De Psicología, 30(2), 463-473. Doi: http://doi.org/10.6018/analesps.30.2.155471

Gaete, R. (2014). Reflexiones sobre las bases y procedimientos de la Teoría Fundamentada. Ciencia, docencia y tecnología, 48, 149-172. Recuperado de: http://www.redalyc.org/pdf/145/14531006006.pdf

Gaitán, A., \& Rey, C. A. (2013). Diferencias en funciones ejecutivas en escolares normales, con trastorno por déficit de atención e hiperactividad, trastorno del cálculo y condición comórbida. Avances en Psicología Latinoamericana, 31(1), 71-85. Recuperado de: $\mathrm{http} / / / \mathrm{www}$. scielo.org.co/pdf/apl/v31n1/v31n1a06.pdf

García-Molina, A., Tirapu-Ustárroz, J., Luna-Lario, P., Ibáñez, J., \& Duque, P. (2010). ¿Son lo mismo inteligencia y funciones ejecutivas? Revista de Neurolología, 50(12), 738-746. Recuperado de: https://www.neurologia.com/articulo/2009713

Gioia, G., Isquith, P., Guy, S., \& Kenworthy, L. (2017). BRIEF-2 Evaluación conductual de la función ejecutiva (M. Maldonado, M. Fournier, R. Martínez-Arias, J. González-Marques, J. Espejo-Saavedra \& P. Santamaría, adaptadores). Madrid: TEA Ediciones.

Goldberg, E. (2001). The executive brain. Frontal lobes and the civilized mind. Oxford: University Press.

Gonzales, F. (1999). Cualitativa en psicología. Rumbos y desafíos. Sao Paulo: Educ.

Goleman, D. (2000). Inteligencia Emocional. Barcelona: Kairos

Hackman, D. A., \& Farah, M. J. (2008). Socioeconomic status and the developing brain. Trends in Cognitive Sciences, 13, 65-73. Doi: https://doi.org/10.1016/j.tics.2008.11.003

Heaven, P. C., \& Ciarrochi, J. (2012). When IQ is not everything: Intelligence, per-sonality and academic performance at school. Personality and Individual Differences, 53(4), 518-522. Doi: https://doi.org/10.1016/j.paid.2012.04.024

Herrnstein, R.J. \& Murray, C. (1994). The bell curve: Intelligence and class structure in American life. New York, NY: Free Press.

Heyder, K., Suchan, B., \& Daum, I. (2004). Cortico-subcortical contributions to executive control. Acta Psychologica, 115, 271-289. Doi: https://doi.org/10.1016/j.actpsy.2003.12.010

Isaza, G., \& Calle, J. (2016). Un acercamiento a la comprension del perfil de la inteligencia emocional. Revista latinoamerica de ciencias sociales, niñez y juventud, 14(1), 331-345. Doi: http://doi.org/10.11600/1692715x.14122220814

Jacobson, L. A., Williford, A. P., \& Pianta, R. C. (2011). The role of executive function in children's competent adjustment to middle school. Child Neuropsychology, 17(3), 255-280.

Doi: http://doi.org/10.1080/09297049.2010.535654

Jensen, A.R. (1973). Educability and Group Differences. London: Methuen.

Krumm, G., Arán Filippetti, V., \& Gutierrez, M. (2018). The contribution of executive functions to creativity in children: What is the role of crystallized and fluid intelligence? Thinking Skills and Creativity, 29, 185-195. Doi: http://doi.org/10.1016/j.tsc.2018.07.006

Losada, L., \& López, R. (2003). Métodos de investigación en ciencias humanas y sociales. Madrid: Paraninfo.

Marambio, K., Gil de Montes, L., Valencia, J., \& Zubieta, E. (2015). Representaciones sociales de inteligencia y los valores culturales que las enmarcan. Psicoperspectivas, 14(3), 4555. Doi: http://doi.org/10.5027/psicoperspectivas-Vol14-Issue3-fulltext-641

Martins, E. C., Osório, A., Veríssimo, M., \& Martins, C. (2016). Emotion understanding in preschool children: The role of executive functions. International Journal of Behavioral Development, 40(1), 1-10. Doi: http://doi.org/10.1177/0165025414556096

Mesa, C. (2018). Caracterización de las inteligencias múltiples de estudiantes de 2 do año de la carrera de Medicina. Revista Médica Electrónica, 40(2), 298-310. Recuperado de http://www.medigraphic.com/pdfs/revmedele/me-2018/me182g.pdf

Miyake, A., \& Friedman, N. P. (2012). The Nature and Organization of Individual Differences in Executive Functions: Four General Conclusions. Current Directions in Psychological Science, 21(1), 8-14. Doi: http://doi.org/10.1177/0963721411429458 
Miyake, A., Friedman, N. P., Emerson, M. J., Witzki, A. H., Howerter, A., \& Wager, T. D. (2000). The unity and diversity of executive functions and their contributions to complex "frontal lobe" tasks: a latent variable analysis. Cognitive Psychology, 41, 49-100. Doi: https://doi.org/10.1006/cogp.1999.0734

Montoya-Arenas, D. A., Trujillo-Orrego, N., \& Pineda-Salazar, D. A. (2010). Capacidad intelectual y función ejecutiva en niños intelectualmente talentosos y en niños con inteligencia promedio. Universitas Psychologica, 9, 737-747. Recuperado de: http://www.scielo.org.co/pdf/rups/v9n3/v9n3a11.pdf

Noble, K. G., Norman, M. F., \& Farah, M. J. (2005). Neurocognitive correlates of socioeconomic status in kindergarten children. Developmental Science, 8, 74-87. Doi: https://doi.org/10.1111/j.1467-7687.2005.00394.x

Pacheco, V. (2003). La inteligencia y el pensamiento creativo: aportes históricos en la educación. Revista Educación 27(1), 17-26. Recuperado de http://www.redalyc.org/articulo.oa?id=44027103

Pennequin, V., Sorel, O., \& Fontaine, R. (2010). Motor planning between 4 and 7 years of age: Changes linked to executive functions. Brain and Cognition, 74, 107-111. Doi: http://doi.org/10.1016/j.bandc.2010.07.003

Pérez, E., \& Medrano, L. (2013). Teorías contemporáneas de la inteligencia. Una revisión crítica de la literatura. Psiencia. Revista Latinoamericana de Ciencia Psicológica, 5(2), 105118.

Reyes, M., Altamar, P., Aguirre; M., \& Murillo, D. (2014). Bienestar en personas mayores en situación de pobreza: determinantes y significados. Revista de Psicología, 23(2), 101105. Doi: http://doi.org/10.5354/0719-0581.2015.36151

Riggs, N. R., Jahromi, L. B., Razza, R. P., Dillworth-Bart, J. E., \& Mueller, U. (2006). Executive function and the promotion of social-emotional competence. Journal of Applied Developmental Psychology, 27, 300-309. Doi: http://doi.org/10.1016/j.appdev.2006.04.002

Rosas, R; Boetto, C., \& Jordán, V. (2005). Introducción a la psicología de la inteligencia. Santiago: Ediciones Universidad Católica de Chile.

Rosenberg, L. (2014). The Associations Between Executive Functions' Capacities, Performance

Process Skills, and Dimensions of Participation in Activities of Daily Life Among Children

of Elementary School Age. Applied Neuropsychology: Child, 0, 1-9. Doi: http://doi.org/10.1080/21622965.2013.821652

Ruiz, J. (2003). Metodología de la investigación cualitativa. Bilbao: Universidad de Deusto.

San Martín, D. (2014). Teoría fundamentada y Atlas.ti: recursos metodológicos para la investigación educativa. Revista electrónica de investigación educativa, 16(1), 104-122. Recuperado de: https://redie.uabc.mx/redie/article/view/727/891

Sánchez, J. (2008). La infancia en la sociedad del conocimiento. Revista iberoamericana de ciencia tecnología y sociedad, 4(11), 23-43. Recuperado de: https://dialnet.unirioja.es/servlet/articulo?codigo $=3044889$

Sastre-Riba. S. (2006). Condiciones tempranas del desarrollo y el aprendizaje: el papel de las funciones ejecutivas. Revista de Neurología, 42(2), 143-151. Recuperado de http://www.mdp.edu.ar/psicologia/psico/sec-

academica/asignaturas/aprendizaje/Condiciones\%20tempranas.pdf

Shaul, S., \& Schwartz, M. (2014). The role of the executive functions in school readiness among preschool-age children. Reading and Writing, 27, 749-768. Doi: http://doi.org/10.1007/s11145-013-9470-3

St Clair-Thompson, H. L., \& Gathercole, S. E. (2006). Executive functions and achievements in school: Shifting, updating, inhibition, and working memory. The Quarterly Journal of Experimental Psychology, 59, 745-759.Doi: http://doi.org/10.1080/17470210500162854

Stelzer, F., Cervigni, M., \& Martino, P. (2011). Desarrollo de las funciones ejecutivas en niños preescolares: una revisión de algunos de sus factores moduladores. Liberabit, 17(1), 93100. Recuperado de: http://www.redalyc.org/articulo.oa?id=68619288011

Strobel, A., Behnke, A., Gärtner, A., \& Strobel, A. (2019). The interplay of intelligence and need for cognition in predicting school grades: A retrospective study. Personality and Individual Differences, 144, 147-152. Doi: https://doi.org/10.1016/j.paid.2019.02.041 
Thorell, L. B., Veleiro, A., Siu, A. F., \& Mohammadi, H. (2013). Examining the relation between ratings of executive functioning and academic achievement: Findings from a cross-cultural study. Child Neuropsychology, 19, 630-638. Doi: http://doi.org/10.1080/09297049.2012.727792

Toledo, M. I., (2012). Sobre la construcción identitaria. Atenea, (506), 43-56. Doi: https://dx.doi.org/10.4067/S0718-04622012000200004

Vivar, C. G., Arantzamendi, M., López-Dicastillo, O., \& Gordo Luis, C. (2010). La Teoría Fundamentada como Metodología de Investigación Cualitativa en Enfermería. Index de Enfermería, 19(4), 283-288. Recuperado de: http://scielo.isciii.es/scielo.php?script=sci_arttext\&pid=S1132-12962010000300011

Welsh, M. C., Pennington, B. F., \& Groisser, D. B. (1991). A normative-developmental study of executive function: A window on prefrontal function in children. Developmental Neuropsychology, 7, 131-149. Doi: http://doi.org/10.1080/87565649109540483

Zelazo, P.D. (2003). The development of executive function. Monographs of the Society for Research in Child Development, 68, 1-27. Doi: https://doi.org/10.1111/j.15405834.2003.06803001.x

Zelazo, P. D., \& Müller, U. (2002). Executive function in typical and atypical development. In U. Goswami (Ed.), Handbook of childhood cognitive development (pp. 445-469). Oxford, UK: Blackwell. Doi: https://dx.doi.org/10.1111\%2Fjcpp.12458 


\section{Anexo 1}

\section{Guión de Entrevista Estructurada}

Fecha:

Nombre del Entrevistado:

Edad:

Colegio:

\section{OBJETIVO GENERAL:}

Conocer las concepciones que niñas y niños, de edades comprendidas entre 8 y 12 años, otorgan al concepto de inteligencia, las características que le atribuyen y la valoración que hacen de las propias capacidades intelectuales.

Tópicos de estudio:

- Concepciones otorgadas al concepto de inteligencia.

- Características atribuidas al desempeño inteligente.

- Valoración de las propias capacidades intelectuales.

Indicaciones Relevantes: junto con saludar y agradecer la participación del niño/niña, contextualizar la actividad entregando coordenadas generales del trabajo y situando esta técnica de recopilación de información (aquí es preciso informar que se realizará una grabación de voz). Luego preguntar explícitamente al niño/niña si quiere participar de la actividad, no obstante, al consentimiento ya entregado por los padres, si no asiente al trabajo propuesto, agradecer su sinceridad y dejar que continúe con sus actividades; en el caso que adhiera proseguir con las preguntas en el mismo orden en la que se proponen.

\section{PREGUNTAS:}

1. Si tuvieras que definir el concepto de inteligencia ¿cómo lo harías? ¿qué es inteligencia?

2. ¿Cuáles son las características que observas en un niño o niña inteligente?

3. ¿Te consideras un niño o niña inteligente? ¿por qué? 